\title{
A New Method of Point-Clouds Accurate Measurement and Reconstruction
}

\author{
Kun Zhang ${ }^{1}$, Weihong $\mathrm{Bi}^{2}$ and Xinghu $\mathrm{Fu}^{3}$ \\ ${ }^{1}$ Yanshan University, Hebei University of Science \& Technology \\ ${ }^{2}$ College of Information Science \& Engineering, Yanshan University \\ euphkun@163.com,bwhong@ysu.edu.cn and fuxinghu@ysu.edu.cn
}

\begin{abstract}
With the reverse engineering development, the accuracy of system is more important in reconstruction, especially in non-contacting measurement. This paper provides a new method measuring the accuracy the point clouds, define a image probability and the point probability according to uncertainty data. The quantity of the uncertain point data is important to measuring the result of reconstruction. The prior data can be catch from the last measurement process, especially the edge data or characteristic points. Referring to prior data, basing on the Bayesian theory the more accuracy posterior data can be computed in this paper. We divided the point cloud into different areas, and organized the data with hierarchical tree-structure. According to the probability of one tree node, we adjust the area corresponding to the node. At last, by using the existing experimental equipment, we verify the measurement of point cloud accuracy algorithm. The depth data was obtained by a laser scanner---SICK LMS100. The depth data can be computed as point data with uncertainty. The result of the reconstruction deeply relies on the quality of prior data.
\end{abstract}

Keywords: reverse engineering; uncertainty data; image probability; accuracy of point cloud measurement

\section{Introduction}

At present, surface reconstruction of point clouds is a hot area of research in computer graphics, virtual reality and reverse engineering. Because of the quality of the point clouds reconstruction affected the presentation of the geometry, it is important to visual calculating. Up to now, a variety of techniques have been proposed, which has different emphasis during the process of reconstruction. For example, Voronoi / Delaunay techniques focus on topology of the reconstruction. Their method gives a minimum sampling density dependent on the local feature size. Besides, there are many different methods, using implicit functions, or RBF etc. This paper has grouped these techniques into two different categories. 1) parametric curves interpolate: parametric curves and surfaces are usually applied to interpolate, approximate and fit the point cloud data. Among this kind method, piecewise linear interpolation is more popular because of its simplicity. People usually choose the linear interpolation to reconstruction the surface. 2) The other class of surface reconstruction techniques is to use implicit functions to represent the unknown free-form surface. In general, these methods will get the zero level set to approximate the initial data set. The kind of implicit functions can conveniently 
reconstruct the complicated surface, especially to the uncompleted surface or noisy data set. Reference [1-7] has been proved the effectiveness of the ability to get the complicated surface by implicit functions. Of course, for high-quality result, references [8-10] have provided some strategies to high-performance process. For example, Reference [8] improved the image process by GPU, and the reference [9] provided an efficient image management system.

However, all of the methods have not considered the uncertainty of the raw data. The errors will be magnified in the final result. Especially the data is measured by mobile laser scanner, like our experiment, the error may be intolerable. So, we are in urgent need of a way to decreasing the error that can decrease the uncertainty of the data, especially a way to measure the uncertainty. Of course, many researchers consider the uncertainty during the reconstruction. For example, $[11,12]$ regarded uncertainty as a parameter tolerance. [13] proposed a method to define the position uncertainty of point clouds. [14] analyzed the shape uncertainty of the point clouds, and provided confidence map and likelihood map. [15] Described a Bayesian model for point cloud uncertainty for surface reconstruction.

These models have been considered the uncertainty of the raw data. But it still existed inevitable defects. From now on, most of them can resolve the uncertainty question by analyzing the imprecise parameter. Of course imprecise parameter is an important factor to the uncertainty. However, there are lots of other factors can interfere with it, for example, a fringe of missing data or measurement data under the harsh environment. Besides, the process computing of uncertainty is closely related to the data of neighboring points. So if we want to get the precise data, there will be exist enormous computation. Maybe some engineers really do not need the high resolution graphic, so they can not suffer the huge computation, because of the wasting of resources and time. That is, the other biggest thing is get the quantifiable terms of the uncertainty, and then we need to measurement the uncertainty during the process of reconstruction.

For the reconstruction of scattered point cloud data, the difficulty lies in the quality and the speed of the results. We can't get a method with high speed and high degree of precision reconstruction. So it is difficult to find the optimal algorithm. But we satisfied with the method, which can meet the needs of engineering precision and speed. Of course that is important to our research.

This paper presented a preliminary discussion of the above problems. Firstly, we quantified the uncertain data by probabilistic models. Then, using the Bayesian theory, we can deal with the raw imprecise data, and get more accuracy data. That is more significant. In contrast with other Bayesian methods, we take the graphic as a whole graphic [16, 17]. Finally, we proved the feasibility of this method by experiments.

\section{The Basic Principle of Uncertain Point Measurement}

In this section, we will discuss the details of definition of uncertain point cloud data model, and introduce the method of judging the precision of the raw point data. Reference [12] firstly provided the reconstruction tactics for disorder point clouds basing on Bayesian statistic. Since that, many researches have been done by scholar, for example, in machine vision, or 3D reconstruction. But little mention has been made of the measurement of accurate. This paper gives a novel precision measurement method based on Bayesian statistic. 
Inspired by uncertain data, we take the whole image as the complete event. Firstly, the image is necessary to discretize at the degree $f$, and the $f$ is the basic cell for the discretization. After discretization, the whole image becomes an order point cloud defined it as $\mathrm{S}$, so $\mathrm{S}$ is a real world for the image in probabilistic. $\mathrm{P}(\mathrm{I})$ can describe the probability $\mathrm{S}$. the all points of $\mathrm{S}$ will be taken as an event in probabilistic. If $\mathrm{D}$ belongs to $\mathrm{S}$, that is $\mathrm{D}$ as one of the point of $\mathrm{S}$. Then the $\mathrm{D}$ is composed by the world coordinate $\mathrm{x}, \mathrm{y}$ and $\mathrm{z}$, that is $\mathrm{D}(\mathrm{x}, \mathrm{y}, \mathrm{z})$.

Assumed that the measurement data are independent, so the union probability of $\mathrm{D}_{\mathrm{m}}$ and $\mathrm{D},\left(D_{m} \wedge D_{n}\right)=P\left(D_{m}\right) \times P\left(D_{n}\right) \quad$. The definition of the point probability is different from the others, because that it is focused on the quality of the measurement data.

Definition 1 : (the image probability) Assumed that the image of object "m", "m" is discretized at the degree $f$, then, you can get the order point cloud $S$, and $S$ is composed of every point $\mathrm{s}_{\mathrm{i}},(\mathrm{i}=1 . . \mathrm{n})$, so we get the probability of $\mathrm{S}$ as follow equation 1 .

$$
P(\Omega)=P(S)=\sum_{\left\{s_{i} \in S\right\}} P\left(S_{i}\right)
$$

By the definition 1, the discretization of image will be seen as the complete event. That will be provided just for the accurate measurement. During the actual measurement, because of time consuming, we will not to get the image of object, and we can take another approximate method to instead it. This method will be introduced in next section.

The definiton 1 describes a probabilistic model of the whole object. The next most important step for us is to define the novel point probability for uncertain data of point. Before explanation the definition of point probability, the two different categories uncertain data will be discussed. Generally, we take the precision, accuracy and error of data as uncertainty. In the point of view of probabilistic, the uncertainty of the data will be concerned as a whole, not analyzing each point of the data. Of course, the efficiency of this method has been greatly improved. As everyone knows, the larger deviant data should produce low probability. Instead, the little deviant data will be exist high probability.

In this paper, the uncertainty will be divided into two categories.

1) We defined one category of the uncertainty data as fake data. Due to the artificial or mechanical error, we get some fake data. Usually, the fake data need to be deleted. In the next process of reconstruction this kind of uncertainty data dose not involved into it.

2) As another category uncertainty, relative to fake data, we call it as inaccurate data. The inaccurate data is the real measure data. However, there exist systematic errors or interference. The measure data often deviate from real image.

Inspired of the KNN algorithm, the whole image will be divided into n-dimension, $\left(\mathrm{K}_{1} \ldots \mathrm{K}_{\mathrm{n}}\right)$, then the probability of $\mathrm{K}_{\mathrm{i}}$ will be computed by eq1, as follows equation 2 .

$$
P(\Omega)=\sum_{\mathrm{i}=1 \ldots \mathrm{n}} P\left(\mathrm{~K}_{i}\right)=\sum_{\mathrm{i}=1 \ldots \mathrm{n}} \sum_{S \in K_{\mathrm{i}}} P(S)
$$

In equation2, the point set $\mathrm{S}$ belong to one of dimension $\mathrm{K}$. So different from the whole image probability, you can get the probability of dimension Ki. Obviously, the image probability depends on each dimension K's probability $\left(P\left(\mathrm{~K}_{i}\right)\right)$. By the way, the $\mathrm{K}$ 's probability $\left({ }^{P\left(K_{i}\right)}\right)$ depends on each point's probability (K's probability $\left({ }^{P\left(K_{i}\right)}\right)$ ). Next, the definition 2 will provide a principal to acquire the probability of point. 
Definition 2: (the point probability) Assumed dimension $\mathrm{Ki}$, if the point $\mathrm{P} \in \mathrm{K}_{\mathrm{i}}$, the probability $\mathrm{P}$ should be the $\mathrm{P}_{\max }$, that is $\mathrm{P}\left(\mathrm{S}_{\mathrm{i}}\right)$ and the $\mathrm{S}_{\mathrm{i}} \in \mathrm{K}_{\mathrm{i}}$. If the point $\mathrm{P} \notin \mathrm{K}_{\mathrm{i}}$, the point $P_{j}$ will be taken as the projection of $P$ on the nearest plane, which consisted by three nearest points from $\mathrm{P}$ to the points set $\mathrm{S}$. So, the probability of the point $\mathrm{P}$ can be presented as the equation 3

$$
P=P_{\max } / \operatorname{dis}\left(P, P_{j}\right) \quad(\text { eq3) }
$$

According to the new definition of probability, we can measure quality of the point cloud. Firstly, you need to discretize the image. In the world coordinate system, the object within maximum $\mathrm{x}$ coordinates $\max (\mathrm{x})$ and minimum $\mathrm{x}$ coordinates $\min (\mathrm{x})$ can be discretized with finite different points.

Besides, we need to discretize it at the degree $\mathrm{f}$, and then the process of the discretization can be handled. Assumed the lowest point cover Cmin. The next equation can be got.

$$
C_{\min }=\frac{H t}{f(\max (x)-\min (x))} \quad \mathrm{eq}(4)
$$

In the eq(4), " $\mathrm{t}$ " stand for the time device takes during runing, and the frequency of the device is $\mathrm{H}(\mathrm{Hz})$.

Actually, the number of the data is $\mathrm{N}, N=3 \times H t$.If the measurement point cover $\alpha<C_{\min }$, the point $\mathrm{S}_{\mathrm{i}}$ and all $\mathrm{S}_{\mathrm{i}}$ belongs to per unit area, the point probability of $\left.\mathrm{S}_{\mathrm{i}},{ }^{P}{ }_{i}\right)=0$. Of course, the missing point can be measured by the discretization. Besides, the degree $\mathrm{f}$ can be defined by user. Usually, we defined the $\mathrm{f}$ by the device parameter.

\section{The Basic Principle of Reconstruction Algorithm}

In this part, a new point clouds reconstruction algorithm will be provided basing the accurate measurement standard in the previous section. Using Bayesian model, the edge point is important to us. We usually take some lower probability areas as the edge points. Inspired by Delaunay algorithm, during reconstruction, we take a line to express a non-edge point.

Assumed that prior data $A=\left\{a_{1} \cdots a_{n}\right\}$, the probability of the data is $\mathrm{P}(\mathrm{A})$; and the posterior data $B=\left\{b_{1} \cdots b_{n}\right\}$; the uncertainty factor c. However, limited by the experimental condition, we actually don't get the real image data. So, we get the prior data $\mathrm{A}$ by the first measurement at $\mathrm{t}_{0}$ moment. Considering the uncertainty $\mathrm{c}$ the prior data $\mathrm{a}_{\mathrm{i}} \in \mathrm{A}$ will be taken as $a_{i}=\left(a_{i}-c, a_{i}+c\right)$. We supposed that the data in range $\left(a_{i}-c, a_{i}+c\right)$ at a uniform distribution. So, according to Bayesian estimation theory, we can get the probability $P(x \mid A)$ as (eq5), the $\mathrm{x}$ shows the difference of prior data and posterior data, $\mathrm{x}=\mathrm{b}-\mathrm{a}$. 


$$
\begin{gathered}
P(x \mid A)=\frac{P(A / x) P(x)}{P(A)}=\frac{P(A \mid x) P(x)}{\int P(A \mid x) P(x) d x} \text { (eq5) } \\
P(A \mid x)=P\left(a_{1} \cdots a_{n} \mid x\right)=\prod_{i=1}^{n} P\left(a_{i} \mid x\right) \text { (eq6) } \\
P(x \mid A)=\frac{P(A \mid x) P(x)}{P(A)}=\frac{\prod_{i=1}^{n} P\left(a_{i} \mid x\right) P(x)}{\int \prod_{i=1}^{n} P\left(a_{i} \mid x\right) P(x) d x}(\text { eq7) }
\end{gathered}
$$

The likelihood function: $L_{n}=P(A \mid x)=\prod_{i=1}^{n} P\left(a_{i} \mid x\right)$

The mathematics expectation function:

$$
x=E(x)=\int x P(x \mid A)=\frac{\int x L_{n} P(x) d x}{\int L_{n} P(x) d x} \text { (eq9) }
$$

The value $\mathrm{x}$ is important in uncertainty measurement, and it also is effective value to evaluate the results of reconstruction. The eq(7) can infer the probability of $\mathrm{x}$, depending on $P(x \mid A)$. Next, the x's probability is can be used for reconstruction.

\subsection{Partition Algorithm —KD-tree-alike}

Likely to KD-tree, the partition algorithm will be divided into different areas. The next work will be based on the partition algorithm. Firstly, the whole point cloud will be taken as a cube. The cube can be divided into smaller different cubes. According to the eq2, the probability of the cube will be computed. Secondly, given a threshold of the probability, the cube will be adjusted. That is the point adjusted by cube's probability. All cube's probability will be adjusted into a limited range. The different cubes can be organized by KD-tree-alike. Finally, by partition algorithm, the organized point areas with similar probability will be computed.

Table 1. The Algorithm of KD-tree-Alike

\begin{tabular}{l}
\hline Algorithm PartitionArea(point-cloud.txt) \\
\hline Input: point-cloud.txt \\
Output: Organized division
\end{tabular}




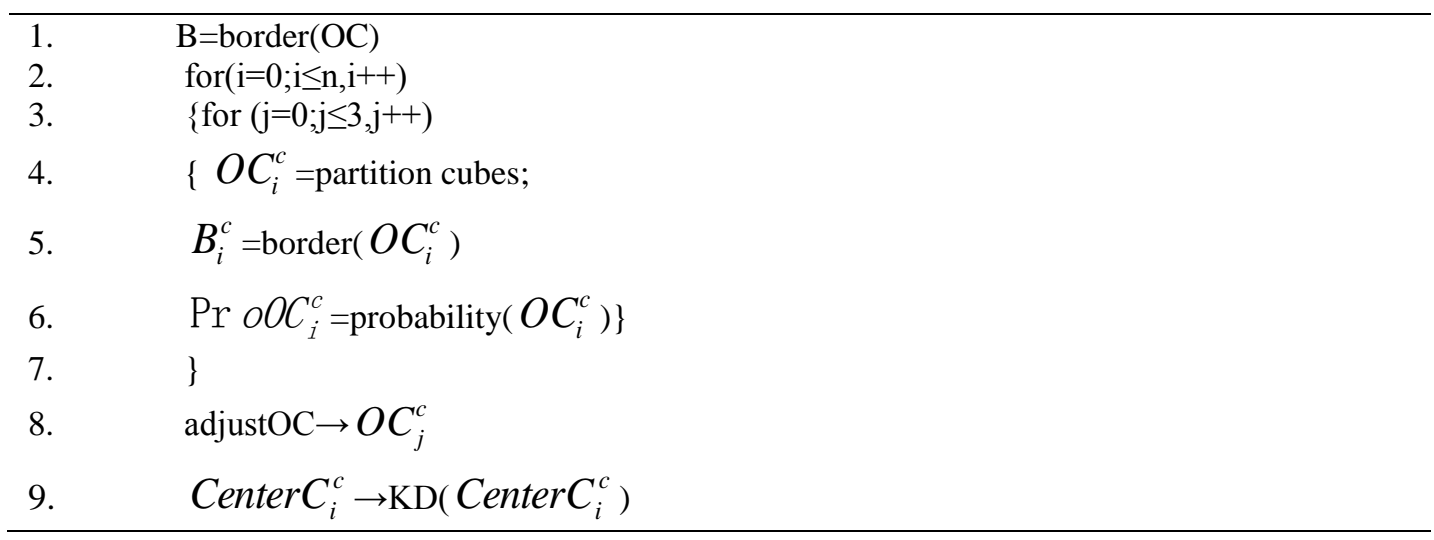

At first, the algorithm import the data of point-cloud.txt into the class $\mathrm{OC}$, and compute the border of the whole view by function $\operatorname{border}(O C)$. Then, the algorithm divided the whole cube into different cubes by function partition cubes at time complexity $\mathrm{O}(\mathrm{n}) . O C_{i}^{c}$ is the children class of $\mathrm{OC}$, it still present a cube composed of point cloud. Of course, using the function border and probability, we can get the border of the cube and the probability of the cube. According to the probability of the cube, we will adjust original $O C_{i}^{c}$ into $O C_{j}^{c}$ by merging cubes with suitable

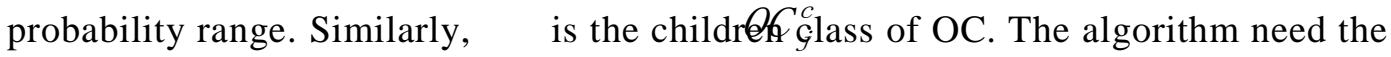
border and the center of $O C_{i}^{c}$.

\subsection{The Edge-point Accurate Computing Algorithm}

Firstly, the edge-point accurate algorithm is important to reduce points by the Bayesian estimation theory. The algorithm gets the entire leaves node in KD-tree and the bigger cubes, in viewing of $\mathrm{AABB}$ algorithm, and picks up the more "collision" cubes. In the next step, the probability of the chosen cubes will be computed, According to eq4, we will adjust the points in the chosen cubes, and get the $\min (\mathrm{E}(\mathrm{x}))$. Finally, the processed points in cube will be computed the probability again, and the algorithm will find the more real edge of the point cloud, that is the conjugated gradient descent.

Table 2. The Algorithm of the Edge-point Accurate Computing

Algorithm EdgeAccurate(the leaves of OC in KD-tree)

Input: $\operatorname{LeafOC}_{i}$ :the leaves of OC in KD-tree

Output: $E C_{i}$ :the edge of the point cloud. 


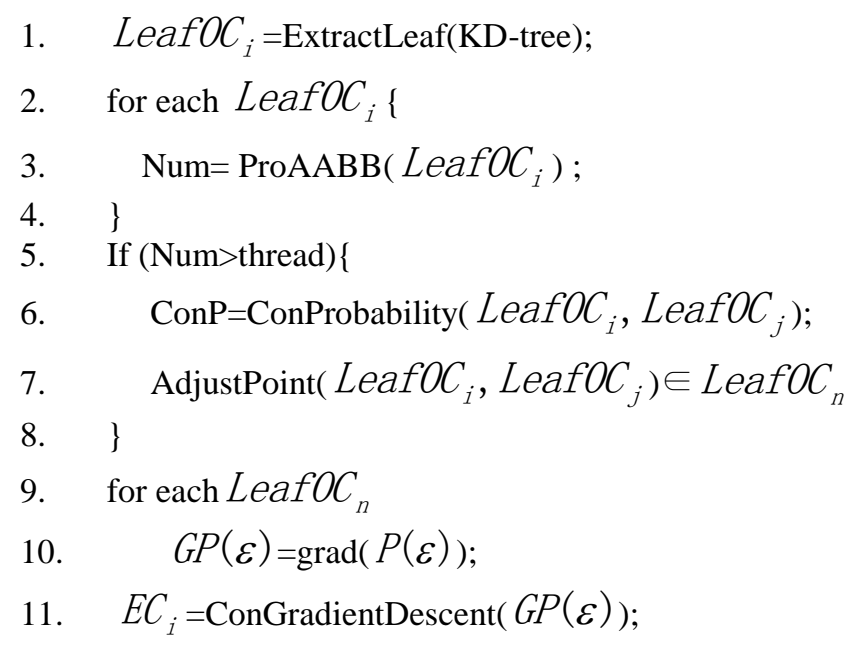

The algorithm of the edge-point accurate computing is to get the edge-points. The input of the algorithm is the leaf node of whole KD-tree, including prior and posterior data, that is $\operatorname{LeafOC}_{i}$. $\mathrm{LeafOC}_{i}$ is the children class if OC. For different KD-trees, LeafOC $_{i}$ will be processed by AABB method. We will pick up the cubes with deep penetrating points. And the variable "num" can present the degree of penetrating points. Given thread with variable "thread", we can pick up cubes which we need. The next, the algorithm will compute the conditional probability of ( ) 1 f last, according to conjugated gradient descent, we can get, that is the edfe of the point cloud, among this, $\varepsilon$ can present the per unit area, so, the $P(\varepsilon)$ is the probability of per unit area $\varepsilon$.

\section{Experiment and Analysis}

In the process of the experiment, one of the important works is to collect the big object data. From now on, there exist two kinds of ways to complete the Laser Data Collection. The first and the most important method is to scan the data by LIDAR. By the LIDAR device, invisible spectrum is provided as measuring medium, especially infrared spectrum. Because of its long wavelength and high safety, infrared spectrum is often used in scanning devices. Hence, the LIDAR scanner with rotary or Linear stepping motor can measure the whole surface of the object. Of course, it provides the raw data of $3 \mathrm{D}$ graphic. The second method for measuring is to scan the data by visible light. Usually, the experiment need CCD(Charge-coupled Device), it can get the visible light. Just because of the limit of the visible light, the distance of the measure is shorter. But by the expensive CCD sensor, the result of the experiment becomes more accurate.

In this paper, data were obtained using a laser scanner---SICK LMS100. SICKLMS100 was produced by SICK Company in Germany. It measured the object using linear LIDAR scanning. LMS100 can provide the more stable and accurate environment. Table 3 describes the parameters of LMS100. 
Table 3. The Parameters of LMS100

\begin{tabular}{|c|c|c|c|c|c|c|c|}
\hline & \multirow{2}{*}{ wavelength } & \multirow{2}{*}{$\begin{array}{l}\text { Measurement } \\
\text { frequency }\end{array}$} & \multirow{2}{*}{$\begin{array}{l}\text { Measurement } \\
\text { distance }\end{array}$} & \multirow{2}{*}{ angle } & \multirow{2}{*}{$\begin{array}{l}\text { Statistical } \\
\text { error }\end{array}$} & \multicolumn{2}{|c|}{$\begin{array}{l}\text { the angular } \\
\text { resolution }\end{array}$} \\
\hline & & & & & & $25 \mathrm{HZ}$ & $50 \mathrm{HZ}$ \\
\hline The Lest & - & $25 \mathrm{HZ}$ & $0.5 \mathrm{~m}$ & - & - & $0.25^{\circ}$ & - \\
\hline Standard & $905 \mathrm{~nm}$ & - & - & - & $\pm 12 \mathrm{~mm}$ & -_ & $0.5^{\circ}$ \\
\hline $\begin{array}{l}\text { The } \\
\text { Most }\end{array}$ & - & $50 \mathrm{HZ}$ & $20 \mathrm{~m}$ & $270^{\circ}$ & $\pm 20 \mathrm{~mm}$ & $0.5^{\circ}$ & - \\
\hline
\end{tabular}

By the LMS100, we can collect the surface of the object. At first, the device will emit a signal to the measured object, which is emitted by a laser pulse, and then it will get through the rotating prism toward the object, at last the signal is reflected to the device. The device will record the whole time of the signal occupied in the process. The device-LMS100 can provide a method to linear measure data. The process of the measuring will be operated with the origin at the center of the device. Then, the measure distance can be got. Assuming light speed c, A and B is the point which we want to measure. And we can get the time $t$ that is the time of back and forth between two points $\mathrm{A}$ and $\mathrm{B}$. Then we can get the $\mathrm{D}$ by the equation $D=C t / 2$, that is the distance between two points $\mathrm{A}$ and $\mathrm{B}$.

LMS100 will be fixed on machine. While the LMS100 working, the parameters of the device will be set up as follows. The range of angle: $75^{\circ} \sim 110^{\circ}$; the angular resolution $0.25^{\circ}$; and the frequency of scanning is $25 \mathrm{HZ}$. Measurement process can be shown in figure:

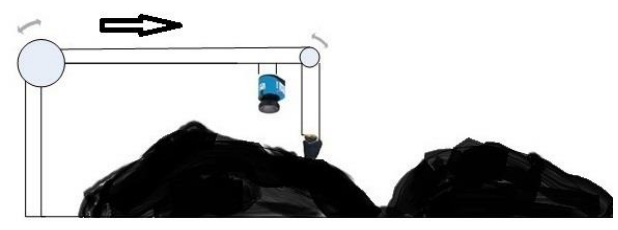

Figure 1. The Present of Experiment Process

\subsection{The Result of Experiment}

By Sick LMS100, this experiment received three times data for the object. The graphic (4-2) is the data that we got. The number of the data is 28341 . It is the distance of the object and the device, and the measuring process takes 8.04(s). The graphic(4-3) is the visualization of the data in graphic(4-2). 


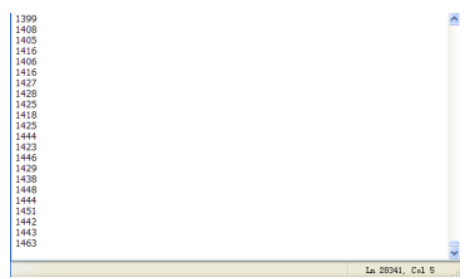

Figure 2. The Data of Experiment

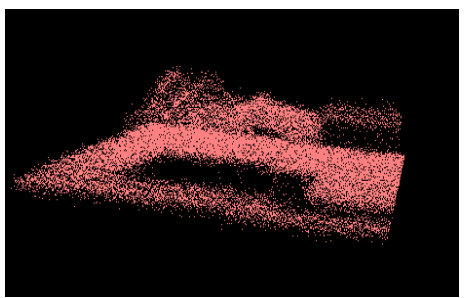

Figure 3. The Visualization of Data

At first, the original data will be simply, as follows. The graphic presents more clear characteristic data. Here, we take the data as prior data. The graphic (4-5) is the simplify the part of graphic(4-4) which is pick out in graphic(4-4) by green curve. It highlighted the most characteristic data. However, these data is the high probability.

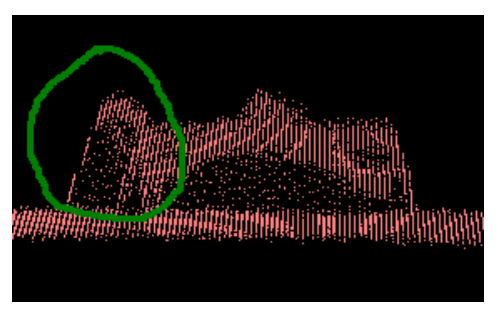

Figure 4. The Simplify of Visualization

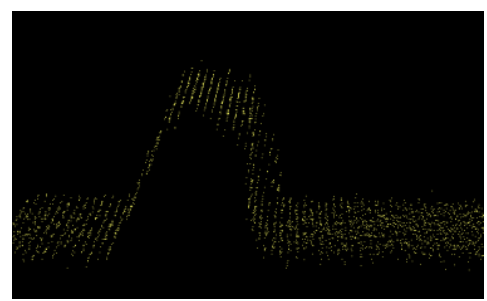

Figure 5. The Detail of Graphic(4-4) 


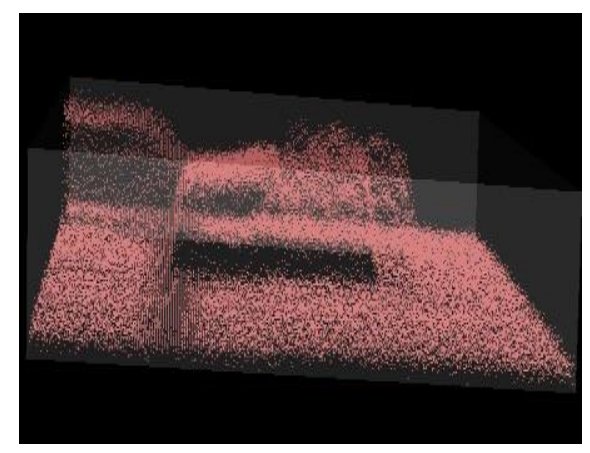

Figure 6. The Edge of Point Cloud

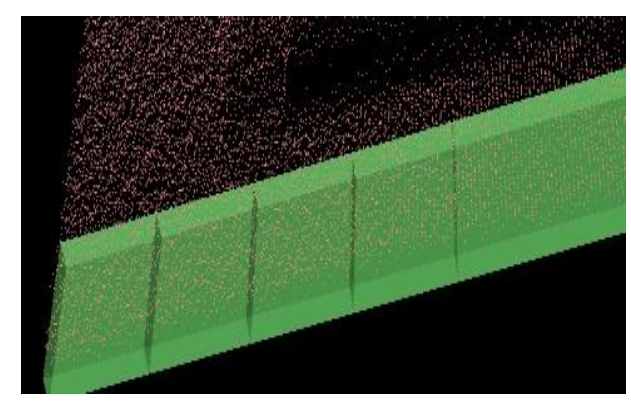

Figure 7. The Present of Point Cloud Organization

We define the simplified data as the symbol--ext(A). Then, the probability of $\operatorname{ext}(\mathrm{A}), \mathrm{P}(\operatorname{ext}(\mathrm{A}))$ is be computed.

The frequency of LMS100 as $\mathrm{H} ; \mathrm{H}=25 \mathrm{~Hz}$

Laser speckle divergent values as $\mathrm{d} ; d=D \times 0.011$

Spot spacing as d'; $d^{\prime}=0.25 \times D=0.322$

The minimum discretization degree:

$$
\begin{aligned}
& \arg \min \left(f_{\text {disperse }}\right) \\
& =48.507 / 25+d+d \\
& =48.507 / 25+28.268+0.3227 \\
& =30.531
\end{aligned}
$$

1) Here, the experiment chooses the discretization degree at $\mathrm{f}$ (disperse) :

$$
\begin{aligned}
& f(\text { disperse })=\arg \min (f(\text { disperse })) \times 5=153 \\
& C_{\min }=\frac{H t}{f(\text { disperse })(\max (x)-\min (x))}=0.01445
\end{aligned}
$$$$
P(\operatorname{ext}(A))=\frac{6308}{28341}=0.2226
$$

2) If the experiment chooses the discretization degree at $\mathrm{f}^{\prime}$ (disperse) :

$$
f^{\prime}(\text { disperse })=\arg \min (f(\text { disperse })) \times 3=91
$$


Then the probability of ext(A):

$$
P^{\prime}(\operatorname{ext}(A))=\frac{8076}{28341}=0.2850
$$

The follows graphic is the amended point data of the graphic (4-9) which have been picked up.

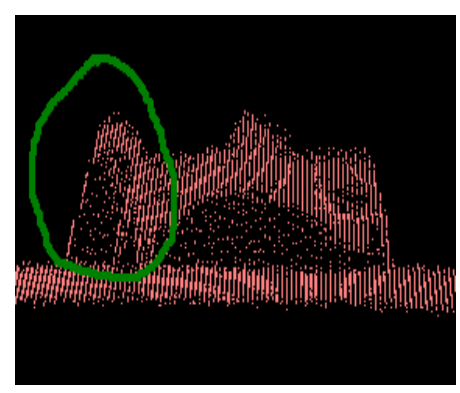

Figure 8. The Simplify of Visualization

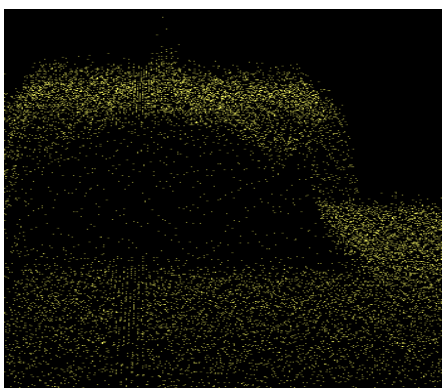

Figure 9. The Detail of Graphic (4-8)

$$
P(B / A)=\frac{\prod_{i=1}^{n} p\left(a_{i} / x\right) p(x)}{\int \prod_{i=1}^{n} p\left(a_{i} / x\right) p(x) d_{x}}=0.4022
$$

\subsection{The Analysis of the Experiment}

The paper provided an algorithm of point cloud accurate reconstruction; it has improved the reliability of the edge-data. What's more, the method of measurement the quality of the point cloud data has been presented in this paper. During refining the edge-data, we provided the partition and accurate edge-data algorithms. The volume of the object is $6.51 \times 10^{-3} \mathrm{~m}^{3}$. The results of different experiments have been shown in Table 4.

Table 4. The Analysis of the Experiment

\begin{tabular}{lccc}
\hline $\mathrm{P}(\operatorname{ext}(\mathrm{A}))$ & $\mathrm{P}(\mathrm{B} / \mathrm{A})$ & volume $\left(10^{-3} \mathrm{~m}^{3}\right)$ & error $(\%)$ \\
\hline 0.2226 & 0.4022 & 6.2098 & 4.61 \\
0.2850 & 0.4189 & 6.235 & 4.22 \\
\hline 0.2226 & 0.5013 & 6.2371 & 4.19
\end{tabular}




\begin{tabular}{lccc}
0.2850 & 0.5127 & 6.25 & 3.99 \\
\hline 0.2226 & 0.5227 & 6.2569 & 3.89 \\
0.2850 & 0.5239 & 6.339 & 2.63 \\
\hline
\end{tabular}

The Table 4 shown the result of threes group experiment, each of them have been measured based on two prior data ext(A). During the experiment, the slower movement speed of the object is; the lager the size of measurement data becomes. The posterior probability of the point cloud is inverse proportional to the volume error. So, the posterior probability can present the accuracy of the point cloud.

\section{Conclusion}

This paper proposed a method of $3 \mathrm{~d}$ laser point cloud accurate measurement and reconstruction. Firstly this paper analyzed the current popular method of point clouds reconstruction, and grouped it in two different categories:1) interpolate,2) implicit functions. Especially, we take the uncertainty of data into count. The formal analysis of the uncertain problems during using the laser point cloud device has been provided. In this paper, probabilistic representation the uncertainty provides an important basis for point cloud reconstruction. Finally using the existing experimental equipment, we get the different accuracy point cloud data, and reconstructed it. By the comparison the "volume", "the prior probabilistic of data" and "the posterior probabilistic of data", we can the proportional relationship between them.

There are many avenues for future work. This paper has analyzed some uncertainty in the process of acquisition of cloud. And in the later calculation process, for example, simplification, registration, matching, recognition, the uncertainty of data will be produced. It will be bring difficulties to the point cloud computing. Finally, we are interested in found the uncertainty and using it to help us reconstruction or computing point cloud, not ignoring the uncertainty.

\section{Acknowledgement}

The paper is funded by Science and Technology Department of Hebei Province in China(No. 14K50120D), and it is sponsored by National Science Foundation of Hebei Province (No. F2013208105) and National Science Foundation of China (No.61272362). It is also supported by Key Research Project for University of Hebei Province(ZD2014029), Hebei Education Department Science Foundation for Young Scholars (QN20131138, QN2014174).

\section{Reference}

[1] N. Shadbolt, T. B. Lee and W. Hall, "The Semantic Web revisited" , IEEE Intelligent Systems, vol. 21, no. 3,(2006), pp. 96-101.

[2] F. Manola and E. Miller, "RDF Primer", W3C Recommendation, http://www.w3.org/TR/2004/REC-rdfprimer-20040210/, (2004).

[3] B. He, M. Patel, Z. Zhang and K. Chang, "Accessing the deep Web", Communications of the ACM, vol. 50, no. 5,(2007), pp. 94-101.

[4] S.S. Sahoo, W. Halb, S. Hellmann, K. Idehen, T. Thibodeau Jr, S. Auer, J. Sequedaand A. Ezzat, "A Survey of Current Approaches for Mapping of Relational Databases to RDF", W3C RDB2RDF Incubator Group, http://www.w3.org/2005/Incubator/rdb2rdf/RDB2RDF_SurveyReport.pdf, (2009).

[5] D.E. Spanos, P. Stavrou and N. Mitrou, "Bringing relational databases into the semantic web: a survey", Semantic Web Journal, vol. 3, no. 2, (2012), pp. 169-209. 
[6] F. Michel, J. Montagnatand C. Faron, "A survey of RDB to RDF translation approaches and tools", Tech. Report \# hal-00903568, version 2, http://hal.archives-ouvertes.fr/hal-00903568, (2014).

[7] E. Prud'hommeaux and M. Hausenblas, "Use Cases and Requirements for Mapping Relational Databases to RDF", W3C Working Draft, http://www.w3.org/TR/rdb2rdf-ucr/, (2010).

[8] M. Arenas, A. Bertails, E. Prud'hommeaux and J. Sequeda, "A Direct Mapping of Relational Data to RDF”, W3C Recommendation, http://www.w3.org/TR/rdb-direct-mapping/, (2012).

[9] S. Das, S. Sundara and R. Cyganiak, "R2RML: RDB to RDF Mapping Language", W3C Recommendation, http://www.w3.org/TR/r2rml, (2012).

[10] B. V. Terrazas and M. Hausenblas, "RDB2RDF Implementation Report", W3C Working Group Note, http://www.w3.org/TR/rdb2rdf-implementations/, (2012).

[11] S. Zhou, Z. Xu, Y. Ni and H. Zhang, "R2RML processor for materializing RDF view of relational data: algorithms and experiments", Proceedings of the 10th Web Information System and Application Conference, Yangzhou, China, IEEE Computer Society, (2013),pp.450-455.

[12] L. E. T. Neto, V. M. P. Vidal, M. A. Casanova and J. M. Monteiro, "R2RML by assertion: a semiautomatic tool for generating customised R2RML mappings", The Semantic Web: ESWC Satellite Events, LNCS, vol. 7955, (2013), pp 248-252.

[13] M. Kifer and H. Boley (Eds), "RIF Overview (Second Edition)", W3C Working Group Note, http://www.w3.org/TR/rif-overview/, (2013).

[14] A. Malhotra, "W3C RDB2RDF Incubator Group Report", W3C Incubator Group Report, http://www.w3.org/2005/Incubator/rdb2rdf/XGR-rdb2rdf, (2009).

[15] C. Marie, G. Hallmark and A. Paschke, "RIF Production Rule Dialect (Second Edition)", W3C Recommendation,http://www.w3.org/TR/rif-prd/, (2013).

[16] M. Hert, G. Reif and H. C. Gall, "A comparison of RDB-to-RDF mapping languages", Proceedings of the 7th International Conference on Semantic Systems, Graz, Austria,(2011), pp. 25-32.

[17] J. Sequeda, S. H. Tirmizi, O. Corcho and D. P. Miranker, "Survey of directly mapping SQL databases to the semantic web", Knowledge Engineering Review, vol. 26, no. 4, (2011), pp. 445-486.

[18] C. Bizer and R. Cyganiak, "D2RQ - lessons learned", Position paper for the W3C Workshop on RDF Access to Relational Databases, Cambridge, USA, http://www.w3.org/2007/03/RdfRDB/papers/d2rqpositionpaper/, (2007).

[19] V. Eisenberg and Y. Kanza, "D2RQ/update: updating relational data via virtual RDF", Proceedings of the 21st World Wide Web Conference (Companion Volume), Lyon, France, (2012), pp. 497-498.

[20] F. Cerbah, "Learning highly structured semantic repositories from relational databases: the RDBToOnto tool", The Semantic Web: Research and Applications: 5th European Semantic Web Conference, ESWC 2008, Tenerife, Canary Islands, Spain, LNCS 5021, Springer Berlin Heidelberg (2008), pp. 777-781.

[21] S. Auer, S. Dietzold, J. Lehmann, S. Hellmann and D. Aumueller. "Triplify: light-weight linked data publication from relational databases", Proceedings of the 18th International Conference on World Wide Web, Madrid, Spain, (2009), pp. 621-630

[22] O. Erling and I. Mikhailov, "Virtuoso: RDF support in a native RDBMS", in Semantic Web Information Management, (2010), pp. 501-519.

[23] J. Sequeda and D. Miranker, "Ultrawrap: SPARQL execution on relational data", Web Semantics: Science, Services and Agents on the World Wide Web,vol. 22, (2013), pp. 19-39.

[24] E. Prud'Hommeaux, "A. Seaborne, SPARQL query language for RDF", W3C Recommendation, http://www.w3.org/TR/rdf-sparql-query, (2008). 
International Journal of Database Theory and Application Vol. 7, No. 6 (2014) 